
\title{
R Research Souture \\ Factors Influencing the Stages of Frailty among Korean Older Adults Focusing on Objective and Subjective Social Isolation
}

\author{
Song Yi Han \\ Sunmoon University \\ Hye Young Jang \\ Hanyang University \\ Young Ko ( $\square$ moodory@gmail.com ) \\ Gachon University
}

\section{Research Article}

Keywords: older adults, frailty, social isolation

Posted Date: May 21st, 2021

DOI: https://doi.org/10.21203/rs.3.rs-537880/v1

License: @ (i) This work is licensed under a Creative Commons Attribution 4.0 International License. Read Full License 


\section{Abstract}

Background: Although many studies have investigated the factors influencing frailty, studies on factors affecting transition between frailty stages are insufficient. This study was conducted to identify factors influencing the stages of frailty in Korean older adults, focusing on objective and subjective social isolation.

Methods: This study analyzed the data of 10,041 older adults from the 2017 National Survey of Older Koreans. Two multiple logistic regression analyses were performed to identify the factors influencing the frailty stages.

Results: Among Korean older adults, $6 \%$ were in a frail stage, and $42.5 \%$ were in the pre-frail stage. The progression to the pre-frail stage was influenced by sociodemographic and health-related characteristics and by objective and subjective social isolation. Contrastingly, the transition from the pre-frail to frail stage was influenced by factors including objective social isolation, declining hearing, and lack of regular exercise.

Conclusions: In the development of future interventions, researchers should consider differences in factors that influence the stages of frailty among Korean older adults. Interventions that help older people maintain existing social relationships or connect to new social networks can delay the transition from the pre-frail to frail stages.

\section{Background}

As the population ages, frailty has become an important public health issue [1]. Likewise, in South Korea, rapid aging has increased the proportion of frail older adults with consequent social problems [2]. As a biological and physiological change that progresses with aging, frailty is defined as a decline in multiple body systems' reserve $[3,4]$. As the frailty progresses in older adults, multiple functions decline, the ability to maintain daily life independently gradually decreases, and eventually, the frail older adults become dependent on others [5]. Frailty also leads to negative health outcomes such as institutionalization, hospitalization, disability, and mortality $[1,3,6-7]$. The quality of life of the individual deteriorates as a result [9]. Moreover, the burden of care on the family and society increases [10]. Frailty is a continuum of robust, pre-frail, and frail stages, where vulnerability to poor health outcomes increases with progression $[4,11]$. While the way to prevent the transition from the robust or pre-frail to frail stage is important [12], studies on the factors influencing each stage of frailty are lacking.

Various tools are used to measure frailty $[3,13,14]$. One of the most frequently used tools is the frailty phenotype suggested by the Cardiovascular Health Study, which defines frailty in five domains: slowness, weakness, fatigue, low physical activity, and body weight loss [3]. However, limitations have been identified in that this tool is practically difficult to use and does not consider individuals with disabilities. In this respect, the Geriatric Advisory Panel suggested a frailty tool that allows easy measurements for community residents with high availability [15]. In this tool, frailty is defined in the following five domains: fatigue, resistance, ambulation, illness, and loss of weight [15]. Based on this, Jung et al. (2016) created the Korean version of the FRAIL scale and verified its clinical utility and validity [13]. Therefore, in this study, we used the FRAIL scale to measure frailty in older adults living in the community. 
According to previous studies, personal factors such as sociodemographic characteristics (e.g., education level, age, gender, and household income) [12,16-18], regular exercise [4, 17], cognitive functions [19, 20], functional levels, and sensory functions [18] have been identified as the risk factors of frailty. In addition, social factors such as social isolation were important factors progressing frailty [21-23]. Social isolation is multidemensional and can be divided into subjective social isolation and objective social isolation [24]. While the latter indicates the lack or inadequacy of interactions with other people, such as the size of a social network or frequency of contact, the former indicates the quality and perception of the relationships with others [24]. Compared to younger generations, the social networks of older adults are decreased in size [25]. The social network of the elderly is mostly composed of family and long-term friends [26], and the number of people with whom important issues could be discussed continues to decrease [27]. Such an increase in social isolation among older adults is thus predicted to be an important risk factor of frailty [21-23]. However, only a few studies have investigated the correlation between the multidimensional properties of social isolation (subjective and objective social isolation) and the stages of frailty.

Therefore, this study aimed to explore the characteristics of subjective and objective social isolation and their correlation with the frailty stages in older adults residing in the community (Aim 1) and to identify the influence of subjective and objective social isolation on progression to the pre-frail stage and the transition from the pre-frail to the frail stage (Aim 2).

\section{Methods}

\section{Study design}

This study conducted a secondary analysis of the 2017 data from the National Survey of Older Koreans (NSOK) to investigate the factors influencing the stages of frailty in older adults living in community, focusing on objective and subjective social isolation.

\section{Data and ethical considerations}

This study analyzed the 2017 data from the NSOK conducted by the Ministry of Health and Welfare [28]. The NSOK has been conducted every three years since 2008 to study older adults aged $\geq 65$ years in South Korea. In 2017, 10,299 older adults aged $\geq 65$ years in 934 regions were studied. The survey uses nationally representative samples of non-institutionalized Korean older adults aged 65 or over who lived in the community. The data can be obtained from the public data portal (data.go.kr) after the institution's application and subsequent approval [28]. This study was conducted with an exemption of Institutional Review Board (IRB) approval at the author's university (IRB No: 1044396-202102-HR-030-01). The data of 258 individuals with missing values among the 10,299 in the original data set were excluded, and 10,041 older adults were included in the analysis.

\section{Measurements}




\section{Frailty}

Frailty was measured using the FRAIL Scale with the following five domains: fatigue, resistance, ambulation, illness, and loss of weight [15], which has been validated for use in older Koreans [13]. In this study, the five domains of the FRAIL Scale were assessed according to the following criteria. 1) Fatigue: For the question, "Have you experienced a significantly reduced level of activity or drive recently?", a "No" response was given a score of 0 , and a "Yes" response was given a score of 1.2) Resistance: For the question, "How difficult is it for you to climb ten stairs without rest?", a response of "Not difficult at all" or "A little difficult" was given a score of 0 (not difficult), and a response of "Very difficult" or "Too difficult to do" was given a score of 1 (difficult). 3) Ambulation: For the question, "How difficult is it for you to complete one round of a walk in a schoolyard (400 m)?", a response of "Not difficult at all" or "A little difficult" was given a score of 0 (not difficult), and a response of "Very difficult" or "Too difficult to do" was given a score of 1 (difficult). 4) Illness: If a participant had three or fewer diseases diagnosed by a health care professional (e.g., hypertension, diabetes, cancer, chronic bronchitis or pulmonary emphysema, angina or cardiac infarction, other heart conditions, asthma, arthritis, stroke [paralysis or cerebral infarction], and chronic renal disease), a score of 0 was given. If a participant had been diagnosed with four or more diseases, a score of 1 was given. 5) Loss of weight: It was defined as loss of weight when an individual had a loss or gain of $5 \mathrm{~kg}$ without weight control over the previous six months and is underweight with body mass index (BMI) of less than $18.5 \mathrm{~kg} / \mathrm{m}^{2}$. In this case, a score of 1 was given. A score of 0 was given to all other cases. The total score of the above five domains was calculated. A total score of $\geq 3$ indicates frail, 1-2 indicates pre-frail, and 0 indicates a robust health state [29].

\section{Social isolation}

Objective social isolation was measured by combining the frequency of contact with family and contact with friends, neighbors, and acquaintances [24], based on two questions: "How often do you communicate (via phone, mobile message, email, letter, etc.) with a family member living elsewhere?" and "How often do you communicate with a friend, neighbor, or acquaintance?". The scoring was based on a seven-point scale (1 = "never", $2=$ " $1-2$ times a year", $3=$ " $1-2$ times every three months", $4=$ " $1-2$ times a month", $5=$ "at least once a week", 6 = "2-3 times a week", 7 = "nearly every day [more than four times a week]") to categorize the subjects into the following two groups: not isolated (nearly every day [more than four times a week], 2-3 times a week, at least once a week, or 1-2 times a month) and isolated (1-2 times every three months, 1-2 times a year, or never). After regrouping, objective social isolation was categorized into the following four groups: 1) not isolated from family or friends, 2) isolated from family only, 3) isolated from friends only, and 4) isolated from both family and friends. Subjective social isolation was measured based on the question: "With how many family members (parents and siblings), relatives, friends, neighbors, and acquaintances are you intimately close (to share all your thoughts and feelings)?".

\section{Sociodemographic and health-related characteristics}

The sociodemographic factors were measured using self-reported questions: gender, age, education level, annual household income, and living arrangement. Age was divided into a 65-74 years group and a $\geq 75$ 
years group. Education level was reclassified into no formal education, elementary school graduation, and more than middle school graduation. Annual income was divided into quartiles: lowest 25\%, 26-50\%, 51$75 \%$, and highest $25 \%$. Living arrangement was divided into living alone and living with others. Health-related factors including Activities of Daily Living (ADL) and Instrumental Activities of Daily Living (IADL) dependency, cognitive function, vision and hearing sensory, and exercise were assessed. ADL dependency was measured based on the seven items of the Korean Activities of Daily Living (K-ADL). IADL dependency was measured based on the ten items of the Korean Instrumental Activities of Daily Living (K-IDL).

Dependency was assigned if at least one item indicated a need for assistance [30]. Cognitive function was measured using the Mini-Mental State Examination for Dementia Screening tool developed by Kim et al. [31]. This tool consists of 19 items, and the total score is calculated from the sum of all items. Normal and cognitive decline are classified according to the norm score based on age, sex, and education level. The validity and reliability have been verified in previous studies, and the reliability of the tool was Cronbach's alpha $=.81[31]$.

Visual and auditory sensory functions were assessed using questions of discomfort in daily life regardless of the use of assistants such as glasses and hearing aids. The questions were about discomfort while watching television, reading the newspaper, and talking on the phone or with someone next to them. The response of "Not uncomfortable" was categorized as "good" and "Uncomfortable or Very uncomfortable" as "not good". We assessed exercise using two questions: "How many days per week do you exercise?" and "How many minutes do you exercise per day?". Individuals who exercised more than 30 minutes a day and more than 3 times a week were classified as "regular exercise".

\section{Statistical analysis}

SPSS 23.0 was used for all statistical analyses. To analyze characteristics of social isolation and stages of frailty and the correlation between social isolation and the stages of frailty, $\chi^{2}$ test, $t$-test, one-way ANOVA with Scheffe test, and descriptive statistics were used (Aim 1). Two multiple logistic regression analyses were performed to identify the factors influencing the frailty stages, one with the "robust" group as the reference and the other with the "pre-frail" group as the reference (Aim 2). For these analyses, the model was run with five sociodemographic characteristics (gender, age, educational level, annual household income, and living arrangement), six health-related characteristics (ADL dependency, IADL dependency, cognitive decline, vision sensory, hearing sensory, and regular exercise), and two factors of social isolation (objective social isolation and subjective social isolation) in association with the stages of frailty. Odds ratios (ORs) indicated the likelihood of membership in the "pre-frail" group (relative to the "robust" group) and the "frail" group (relative to the "pre-frail" group).

\section{Results}

\section{Differences in stages of frailty according to the characteristics of the subjects}


The general characteristics of the subjects are presented in Table 1. The result showed that $51.5 \%$ of the subjects were in a robust stage, $42.5 \%$ in a pre-frail stage, and $6.0 \%$ in a frail stage. The frailty stages varied according to age, gender, education level, annual household income, and living arrangement, in addition to ADL dependency, IADL dependency, cognitive decline, visual and auditory sensory functions, and objective and subjective social isolation $(p<.001)$ (Table 1$)$.

\section{Table 1}

Stages of frailty according to sociodemographic and health-related characteristics 


\begin{tabular}{|c|c|c|c|c|c|c|c|}
\hline \multirow[t]{3}{*}{ Variables } & \multirow{3}{*}{$\begin{array}{l}\text { Categories } \\
\text { (range) }\end{array}$} & \multirow{3}{*}{\multicolumn{2}{|c|}{ Total }} & \multicolumn{3}{|c|}{ Stages of frailty } & \multirow{3}{*}{$\begin{array}{l}\text { X2 or F } \\
\text { (p) } \\
\text { (Scheffe } \\
\text { test) }\end{array}$} \\
\hline & & & & \multirow{2}{*}{$\begin{array}{l}\text { Robust } \\
\mathrm{n}(\%) \text { or } \\
\mathrm{M} \pm \mathrm{SD}\end{array}$} & \multirow[t]{2}{*}{ Pre-frail } & \multirow{2}{*}{$\begin{array}{l}\text { Frail } \\
\mathrm{n}(\%) \text { or } \\
\mathrm{M} \pm \mathrm{SD}\end{array}$} & \\
\hline & & & & & & & \\
\hline Total & & 10,04 & & $\begin{array}{l}5,171 \\
(51.5)\end{array}$ & $\begin{array}{l}4266 \\
(42.5)\end{array}$ & $604(6.0)$ & \\
\hline \multirow[t]{2}{*}{ Gender } & Male & 4277 & $(42.6)$ & $\begin{array}{l}2526 \\
(48.8)\end{array}$ & $\begin{array}{l}1590 \\
(37.3)\end{array}$ & $161(26.7)$ & \multirow[t]{2}{*}{$\begin{array}{l}194.926 \\
(<.001)\end{array}$} \\
\hline & Female & 5764 & $(57.4)$ & $\begin{array}{l}2645 \\
(51.2)\end{array}$ & $\begin{array}{l}2676 \\
(62.7)\end{array}$ & 443 (73.3) & \\
\hline \multirow[t]{2}{*}{ Age (years) } & $65-74$ & 5841 & $(58.2)$ & $\begin{array}{l}3502 \\
(67.7)\end{array}$ & $\begin{array}{l}2169 \\
(50.8)\end{array}$ & $170(28.1)$ & \multirow{2}{*}{$\begin{array}{l}512.890 \\
(<.001)\end{array}$} \\
\hline & $\geq 75$ & 4200 & $(41.8)$ & $\begin{array}{l}1669 \\
(32.3)\end{array}$ & $\begin{array}{l}2097 \\
(49.2)\end{array}$ & $4359(71.9)$ & \\
\hline \multirow[t]{3}{*}{ Education level } & $\begin{array}{l}\text { No formal } \\
\text { education }\end{array}$ & 2376 & $(23.7)$ & $\begin{array}{l}838 \\
(16.2)\end{array}$ & $\begin{array}{l}1267 \\
(29.7)\end{array}$ & $271(44.9)$ & \multirow{3}{*}{$\begin{array}{l}523.330 \\
(<.001)\end{array}$} \\
\hline & $\begin{array}{l}\text { Elementary } \\
\text { school }\end{array}$ & 3444 & $(34.3)$ & $\begin{array}{l}1700 \\
(32.9)\end{array}$ & $\begin{array}{l}1560 \\
(36.6)\end{array}$ & $184(30.4)$ & \\
\hline & $\begin{array}{l}\geq \text { Middle } \\
\text { school }\end{array}$ & 4221 & $(42.0)$ & $\begin{array}{l}2633 \\
(50.9)\end{array}$ & $\begin{array}{l}1439 \\
(33.7)\end{array}$ & $149(24.7)$ & \\
\hline \multirow{4}{*}{$\begin{array}{l}\text { Household } \\
\text { income } \\
\text { (10,000won/year) }\end{array}$} & Q1 ( $\leq 686)$ & 2489 & $(24.7)$ & $\begin{array}{l}1027 \\
(19.9)\end{array}$ & $\begin{array}{l}1255 \\
(29.4)\end{array}$ & 207 (34.4) & \multirow{4}{*}{$\begin{array}{l}304.215 \\
(<.001)\end{array}$} \\
\hline & $\begin{array}{l}\text { Q2 (687- } \\
991)\end{array}$ & 2528 & $(25.2)$ & $\begin{array}{l}1189 \\
(23.0)\end{array}$ & $\begin{array}{l}1136 \\
(26.6)\end{array}$ & $203(33.6)$ & \\
\hline & $\begin{array}{l}\text { Q3 (992- } \\
1,470)\end{array}$ & 2507 & $(25.0)$ & $\begin{array}{l}1361 \\
(26.3)\end{array}$ & $\begin{array}{l}1037 \\
(24.3)\end{array}$ & $109(18.0)$ & \\
\hline & $\begin{array}{l}\text { Q4 } \\
(\geq 1471)\end{array}$ & 2517 & $(25.1)$ & $\begin{array}{l}1594 \\
(30.8)\end{array}$ & 838 (19.7) & $85(14.0)$ & \\
\hline \multirow[t]{2}{*}{ Living alone } & No & 7636 & $(76.1)$ & $\begin{array}{l}4145 \\
(80.2)\end{array}$ & $\begin{array}{l}3092 \\
(72.5)\end{array}$ & 399 (66.1) & \multirow{2}{*}{$\begin{array}{l}110.756 \\
(<.001)\end{array}$} \\
\hline & Yes & 2405 & $(23.9)$ & $\begin{array}{l}1026 \\
(19.8)\end{array}$ & $\begin{array}{l}1174 \\
(27.5)\end{array}$ & 205 (33.9) & \\
\hline \multirow[t]{2}{*}{$\begin{array}{l}\text { Dependency on } \\
\text { ADL }\end{array}$} & No & 9356 & $(93.2)$ & $\begin{array}{l}5082 \\
(98.3)\end{array}$ & $\begin{array}{l}3946 \\
(92.5)\end{array}$ & $328(54.3)$ & \multirow{2}{*}{$\begin{array}{l}1650.621 \\
(<.001)\end{array}$} \\
\hline & Yes & 685 & $(6.8)$ & $89(1.7)$ & $320(7.5)$ & $276(45.7)$ & \\
\hline \multirow[t]{2}{*}{$\begin{array}{l}\text { Dependency on } \\
\text { IADL }\end{array}$} & No & 7760 & (77.3) & $\begin{array}{l}4621 \\
(89.4)\end{array}$ & $\begin{array}{l}3014 \\
(70.6)\end{array}$ & $125(20.7)$ & \multirow{2}{*}{$\begin{array}{l}1638.457 \\
(<.001)\end{array}$} \\
\hline & Yes & 2281 & $(22.7)$ & $\begin{array}{l}550 \\
(10.6)\end{array}$ & $\begin{array}{l}1252 \\
(29.4)\end{array}$ & $479(79.3)$ & \\
\hline
\end{tabular}




\begin{tabular}{|c|c|c|c|c|c|c|c|}
\hline \multirow[t]{2}{*}{ Cognitive decline } & No & 8360 & (83.3) & $\begin{array}{l}4455 \\
(86.1)\end{array}$ & $\begin{array}{l}3472 \\
(81.4)\end{array}$ & $433(71.7)$ & \multirow{2}{*}{$\begin{array}{l}99.419 \\
(<.001)\end{array}$} \\
\hline & Yes & 1681 & (16.7) & $\begin{array}{l}716 \\
(13.9)\end{array}$ & 794 (18.6) & $171(28.3)$ & \\
\hline \multirow[t]{2}{*}{ Vision } & Good & 6651 & $(66.2)$ & $\begin{array}{l}3768 \\
(72.9)\end{array}$ & $\begin{array}{l}2596 \\
(60.9)\end{array}$ & $287(47.5)$ & \multirow{2}{*}{$\begin{array}{l}251.178 \\
(<.001)\end{array}$} \\
\hline & Not good & 3390 & $(33.8)$ & $\begin{array}{l}1403 \\
(27.1)\end{array}$ & $\begin{array}{l}1670 \\
(39.1)\end{array}$ & $317(52.5)$ & \\
\hline \multirow[t]{2}{*}{ Hearing } & Good & 8249 & $(82.2)$ & $\begin{array}{l}4512 \\
(87.3)\end{array}$ & $\begin{array}{l}3360 \\
(78.8)\end{array}$ & $377(62.4)$ & \multirow{2}{*}{$\begin{array}{l}285.023 \\
(<.001)\end{array}$} \\
\hline & Not good & 1792 & $(17.8)$ & $\begin{array}{l}659 \\
(12.7)\end{array}$ & $906(21.2)$ & $227(37.6)$ & \\
\hline \multirow[t]{2}{*}{ Exercise } & Regular & 5311 & $(52.9)$ & $\begin{array}{l}3212 \\
(62.1)\end{array}$ & $\begin{array}{l}2008 \\
(47.1)\end{array}$ & $92(15.2)$ & \multirow{2}{*}{$\begin{array}{l}578.650 \\
(<.001)\end{array}$} \\
\hline & Irregular & 4730 & $(47.1)$ & $\begin{array}{l}1959 \\
(37.9)\end{array}$ & $\begin{array}{l}2258 \\
(52.9)\end{array}$ & $512(84.8)$ & \\
\hline \multirow[t]{4}{*}{$\begin{array}{l}\text { Objective social } \\
\text { isolation }\end{array}$} & $\begin{array}{l}\text { Not } \\
\text { isolated } \\
\text { from } \\
\text { family or } \\
\text { friends }\end{array}$ & 7559 & (75.3) & $\begin{array}{l}4286 \\
(82.9)\end{array}$ & $\begin{array}{l}2993 \\
(70.1)\end{array}$ & $280(46.4)$ & \multirow[t]{4}{*}{$\begin{array}{l}590.259 \\
(<.001)\end{array}$} \\
\hline & $\begin{array}{l}\text { Isolated } \\
\text { from } \\
\text { family only }\end{array}$ & 597 & (5.9) & $283(5.5)$ & $277(6.5)$ & $37(6.1)$ & \\
\hline & $\begin{array}{l}\text { Isolated } \\
\text { from } \\
\text { friends } \\
\text { only }\end{array}$ & 1626 & $(16.2)$ & $\begin{array}{l}529 \\
(10.2)\end{array}$ & $857(20.1)$ & 240(39.7) & \\
\hline & $\begin{array}{l}\text { Isolated } \\
\text { from both } \\
\text { family and } \\
\text { friends }\end{array}$ & 259 & $(2.6)$ & $73(1.4)$ & 139 (3.3) & $47(7.8)$ & \\
\hline \multirow{3}{*}{$\begin{array}{l}\text { Subjective social } \\
\text { isolation }\end{array}$} & \multirow{3}{*}{$\begin{array}{l}\text { Number of } \\
\text { persons }\end{array}$} & \multirow{3}{*}{\multicolumn{2}{|c|}{$2.28 \pm 2.66$}} & \multirow{3}{*}{$\begin{array}{l}2.76 \pm 2.91 \\
c\end{array}$} & \multirow[t]{3}{*}{$1.83 \pm 2.27^{b}$} & \multirow{3}{*}{$\begin{array}{l}1.29 \pm 2.07 \\
a\end{array}$} & 195.03 \\
\hline & & & & & & & $(<.001)$ \\
\hline & & & & & & & $(a<b<c)$ \\
\hline
\end{tabular}

\section{Differences in social isolation according to the characteristics of the subjects}


Subjective social isolation varied according to gender, age, education level, annual household income, and living arrangement, in addition to ADL dependency, IADL dependency, cognitive decline, and visual and auditory sensory functions (Table 2).

Table 2

Subjective social isolation according to sociodemographic and health-related characteristics 


\begin{tabular}{|c|c|c|c|}
\hline Variables & Categories & $M \pm S D$ & $\begin{array}{l}\text { t or } F(p) \\
\text { (Scheffe test) }\end{array}$ \\
\hline \multirow[t]{2}{*}{ Gender } & Male & $2.34 \pm 2.85$ & \multirow[t]{2}{*}{$2.02(.044)$} \\
\hline & Female & $2.23 \pm 2.51$ & \\
\hline \multirow[t]{2}{*}{ Age (years) } & $65-74$ & $2.63 \pm 2.83$ & \multirow[t]{2}{*}{$16.12(<.001)$} \\
\hline & $\geq 75$ & $1.80 \pm 2.32$ & \\
\hline \multirow[t]{3}{*}{ Education level } & None ${ }^{a}$ & $1.46 \pm 1.89$ & \multirow{3}{*}{$\begin{array}{l}223.29(<.001) \\
(a<b<c)\end{array}$} \\
\hline & Elementary school ${ }^{b}$ & $2.15 \pm 2.43$ & \\
\hline & $\geq$ Middle school $^{c}$ & $2.85 \pm 3.05$ & \\
\hline \multirow[t]{4}{*}{ Household income (10,000won/year) } & $\mathrm{Q} 1(\leq 686)^{\mathrm{a}}$ & $1.77 \pm 2.27$ & \multirow{4}{*}{$\begin{array}{l}92.20(<.001) \\
(a<b<c<d)\end{array}$} \\
\hline & Q2 (687-991) ${ }^{b}$ & $2.07 \pm 2.45$ & \\
\hline & Q3 $(992-1,470)^{\mathrm{C}}$ & $2.32 \pm 2.68$ & \\
\hline & $\mathrm{Q} 4(\geq 1471)^{\mathrm{d}}$ & $2.95 \pm 3.04$ & \\
\hline \multirow[t]{2}{*}{ Living alone } & No & $2.37 \pm 2.73$ & \multirow[t]{2}{*}{$6.39(<.001)$} \\
\hline & Yes & $2.00 \pm 2.40$ & \\
\hline \multirow[t]{2}{*}{ Dependency on ADL } & No & $2.34 \pm 2.65$ & \multirow[t]{2}{*}{$7.63(<.001)$} \\
\hline & Yes & $1.51 \pm 2.73$ & \\
\hline \multirow[t]{2}{*}{ Dependency on IADL } & No & $2.53 \pm 2.75$ & \multirow[t]{2}{*}{$20.13(<.001)$} \\
\hline & Yes & $1.44 \pm 2.11$ & \\
\hline \multirow[t]{2}{*}{ Cognitive decline } & No & $2.35 \pm 2.65$ & \multirow[t]{2}{*}{$5.71(<.001)$} \\
\hline & Yes & $1.94 \pm 2.67$ & \\
\hline \multirow[t]{2}{*}{ Vision } & Good & $2.51 \pm 2.81$ & \multirow[t]{2}{*}{$13.22(<.001)$} \\
\hline & Not good & $1.82 \pm 2.28$ & \\
\hline \multirow[t]{2}{*}{ Hearing } & Good & $2.42 \pm 2.74$ & \multirow[t]{2}{*}{$12.91(<.001)$} \\
\hline & Not good & $1.65 \pm 2.16$ & \\
\hline \multirow[t]{2}{*}{ Exercise } & Regular & $2.66 \pm 2.87$ & \multirow[t]{2}{*}{$15.62(<.001)$} \\
\hline & Irregular & $1.85 \pm 2.33$ & \\
\hline
\end{tabular}




\section{Objective social isolation}

Objective social isolation was different by gender, age, education level, annual household income, living arrangement, ADL dependency, IADL dependency, cognitive decline, and visual and auditory functions (Table $3)$.

Table 3

Objective social isolation according to sociodemographic and health-related characteristics 


\begin{tabular}{|c|c|c|c|c|c|c|c|}
\hline Variables & Categories & $\begin{array}{l}\text { Total } \\
\%\end{array}$ & $\begin{array}{l}\text { Not } \\
\text { isolated } \\
\text { from } \\
\text { family or } \\
\text { friends }\end{array}$ & $\begin{array}{l}\text { Isolated } \\
\text { from } \\
\text { family } \\
\text { only }\end{array}$ & $\begin{array}{l}\text { Isolated } \\
\text { from } \\
\text { friends } \\
\text { only }\end{array}$ & $\begin{array}{l}\text { Isolated } \\
\text { from both } \\
\text { family } \\
\text { and } \\
\text { friends }\end{array}$ & $\begin{array}{l}X 2 \text { or } F \\
\text { (p) }\end{array}$ \\
\hline \multirow[t]{2}{*}{ Gender } & Male & 42.6 & $\begin{array}{l}3189 \\
(42.2)\end{array}$ & $\begin{array}{l}276 \\
(46.2)\end{array}$ & $685(42.1)$ & $127(49.0)$ & \multirow{2}{*}{$\begin{array}{l}8.28 \\
(.041)\end{array}$} \\
\hline & Female & 57.4 & $\begin{array}{l}4370 \\
(57.8)\end{array}$ & $\begin{array}{l}321 \\
(53.8)\end{array}$ & 941 (57.9) & $132(51.0)$ & \\
\hline \multirow[t]{2}{*}{ Age (years) } & $65-74$ & 58.2 & $\begin{array}{l}4614 \\
(61.0)\end{array}$ & $\begin{array}{l}436 \\
(73.0)\end{array}$ & $651(40.1)$ & $140(54.1)$ & \multirow{2}{*}{$\begin{array}{l}300.56 \\
(<.001)\end{array}$} \\
\hline & $\geq 75$ & 41.8 & $\begin{array}{l}2945 \\
(39.0)\end{array}$ & $\begin{array}{l}161 \\
(27.0)\end{array}$ & 975 (59.9) & $119(45.9)$ & \\
\hline \multirow[t]{3}{*}{ Education level } & $\begin{array}{l}\text { No formal } \\
\text { education }\end{array}$ & 23.7 & $\begin{array}{l}1564 \\
(20.7)\end{array}$ & $\begin{array}{l}134 \\
(22.4)\end{array}$ & $596(36.6)$ & $82(31.7)$ & \multirow{3}{*}{$\begin{array}{l}229.61 \\
(<.001)\end{array}$} \\
\hline & $\begin{array}{l}\text { Elementary } \\
\text { school }\end{array}$ & 34.3 & $\begin{array}{l}2652 \\
(35.1)\end{array}$ & $\begin{array}{l}173 \\
(29.0)\end{array}$ & $540(33.2)$ & 79 (30.5) & \\
\hline & $\begin{array}{l}\geq \text { Middle } \\
\text { school }\end{array}$ & 42.0 & $\begin{array}{l}3343 \\
(44.2)\end{array}$ & $\begin{array}{l}290 \\
(48.6)\end{array}$ & $490(30.2)$ & $98(37.8)$ & \\
\hline \multirow{4}{*}{$\begin{array}{l}\text { Household } \\
\text { income } \\
\text { (10,000won/year) }\end{array}$} & Q1 (s686) & 24.7 & $\begin{array}{l}1676 \\
(22.2)\end{array}$ & $\begin{array}{l}189 \\
(31.6)\end{array}$ & $524(32.2)$ & $100(38.6)$ & \multirow[t]{4}{*}{$\begin{array}{l}199.21 \\
(<.001)\end{array}$} \\
\hline & $\begin{array}{l}\text { Q2 (687- } \\
991)\end{array}$ & 25.2 & $\begin{array}{l}1867 \\
(24.7)\end{array}$ & $\begin{array}{l}143 \\
(24.0)\end{array}$ & $427(26.3)$ & $91(35.1)$ & \\
\hline & $\begin{array}{l}\text { Q3 (992- } \\
1,470)\end{array}$ & 25.0 & $\begin{array}{l}1937 \\
(25.6)\end{array}$ & $\begin{array}{l}127 \\
(21.3)\end{array}$ & 398 (24.5) & 45 (17.4) & \\
\hline & $\begin{array}{l}\text { Q4 } \\
(\geq 1471)\end{array}$ & 25.1 & $\begin{array}{l}2079 \\
(27.5)\end{array}$ & $\begin{array}{l}138 \\
(23.1)\end{array}$ & $277(17.0)$ & $23(8.9)$ & \\
\hline \multirow[t]{2}{*}{ Living alone } & No & 76.1 & $\begin{array}{l}5914 \\
(78.2)\end{array}$ & $\begin{array}{l}357 \\
(59.8)\end{array}$ & $1214(74.7)$ & $151(58.5)$ & \multirow[t]{2}{*}{$\begin{array}{l}152.31 \\
(<.001)\end{array}$} \\
\hline & Yes & 23.9 & $\begin{array}{l}1645 \\
(21.8)\end{array}$ & $\begin{array}{l}240 \\
(40.2)\end{array}$ & $412(25.3)$ & $108(41.5)$ & \\
\hline \multirow[t]{2}{*}{$\begin{array}{l}\text { Dependency on } \\
\text { ADL }\end{array}$} & No & 93.2 & $\begin{array}{l}7221 \\
(95.5)\end{array}$ & $\begin{array}{l}563 \\
(94.3)\end{array}$ & 1354(83.3) & $218(84.2)$ & \multirow[t]{2}{*}{$\begin{array}{l}350.97 \\
(<.001)\end{array}$} \\
\hline & Yes & 6.8 & $338(4.5)$ & $34(5.7)$ & $272(16.7)$ & 41 (15.8) & \\
\hline \multirow[t]{2}{*}{$\begin{array}{l}\text { Dependency on } \\
\text { IADL }\end{array}$} & No & 77.3 & $\begin{array}{l}6180 \\
(81.8)\end{array}$ & $\begin{array}{l}477 \\
(79.9)\end{array}$ & $942(57.9)$ & $161(62.3)$ & \multirow[t]{2}{*}{$\begin{array}{l}468.41 \\
(<.001)\end{array}$} \\
\hline & Yes & 22.7 & $\begin{array}{l}1379 \\
(18.2)\end{array}$ & $\begin{array}{l}120 \\
(20.1)\end{array}$ & $684(42.1)$ & $98(37.7)$ & \\
\hline \multirow[t]{2}{*}{ Cognitive decline } & No & 83.3 & $\begin{array}{l}6458 \\
(85.4)\end{array}$ & $\begin{array}{l}502 \\
(84.1)\end{array}$ & $1212(74.6)$ & $188(72.6)$ & \multirow[t]{2}{*}{$\begin{array}{l}135.58 \\
(<.001)\end{array}$} \\
\hline & Yes & 16.7 & $\begin{array}{l}1101 \\
(14.6)\end{array}$ & $\begin{array}{l}95 \\
(15.9)\end{array}$ & $414(25.4)$ & $71(27.4)$ & \\
\hline
\end{tabular}




\begin{tabular}{|c|c|c|c|c|c|c|c|}
\hline \multirow[t]{2}{*}{ Vision } & Good & 66.2 & $\begin{array}{l}5172 \\
(68.4)\end{array}$ & $\begin{array}{l}423 \\
(71.0)\end{array}$ & 909 (55.9) & $147(56.8)$ & \multirow[t]{2}{*}{$\begin{array}{l}110.72 \\
(<.001)\end{array}$} \\
\hline & Not good & 33.8 & $\begin{array}{l}2387 \\
(31.6)\end{array}$ & $\begin{array}{l}174 \\
(29.0)\end{array}$ & $717(44.1)$ & $112(43.2)$ & \\
\hline \multirow[t]{2}{*}{ Hearing } & Good & 82.2 & $\begin{array}{l}6364 \\
(84.2)\end{array}$ & $\begin{array}{l}516 \\
(86.4)\end{array}$ & $\begin{array}{l}1195 \\
(73.5)\end{array}$ & $174(67.3)$ & \multirow[t]{2}{*}{$\begin{array}{l}150.88 \\
(<.001)\end{array}$} \\
\hline & Not good & 17.8 & $\begin{array}{l}1195 \\
(15.8)\end{array}$ & $\begin{array}{l}81 \\
(13.6)\end{array}$ & $431(26.5)$ & 85 (32.7) & \\
\hline \multirow[t]{2}{*}{ Exercise } & Regular & 52.9 & $\begin{array}{l}4258 \\
(56.3)\end{array}$ & $\begin{array}{l}323 \\
(54.2)\end{array}$ & $637(39.2)$ & $93(35.8)$ & \multirow[t]{2}{*}{$\begin{array}{l}189.64 \\
(<.001)\end{array}$} \\
\hline & Irregular & 47.1 & $\begin{array}{l}3301 \\
(43.7)\end{array}$ & $\begin{array}{l}274 \\
(45.8)\end{array}$ & $989(60.8)$ & $166(64.2)$ & \\
\hline
\end{tabular}

\section{Factors influencing the stages of frailty}

The multiple logistic regression analysis showed that the factors influencing the transition from the robust to pre-frail stage differed from those influencing the transition from the pre-frail to frail stage (Table 4). For subjective or objective social isolation, the probability of transition from the robust to pre-frail stage was high. The pre-frail stage was found with low probability in many people in an intimate relationship (OR 0.93, 95\% $\mathrm{Cl}=0.91-0.95$ ). Simultaneously, objective social isolation showed a higher probability of transition from the robust to pre-frail stage. All factors of sociodemographic and health-related characteristics influenced the transition from the robust to pre-frail stage.

In contrast, when the sociodemographic and health-related characteristics were adjusted, only objective social isolation influenced the transition from the pre-frail to frail stage: isolation from family living elsewhere (OR $1.57,95 \% \mathrm{Cl}=1.04-2.39$ ), isolation from friends ( $\mathrm{OR} 1.75,95 \% \mathrm{Cl}=1.39-2.19)$, and isolation from both friends and family (OR $2.56,95 \% \mathrm{Cl}=1.67-3.92)$. The probability of transition from the pre-frail to frail stage was also high in female older adults and in subjects showing ADL dependency, IADL dependency, reduced vision or hearing, and lack of regular exercise.

\section{Discussion}

This study aimed to identify factors influencing the stages of frailty in Korean older adults, focusing on objective and subjective social isolation. The results showed that the transition from the robust to pre-frail stage was affected by both objective and subjective social isolation. Conversely, only objective social isolation influenced the transition from the pre-frail to frail stage. This indicated that social isolation was an important factor in the transition from the robust or pre-frail to the frail stage and that different factors influenced each transition among the stages of frailty.

This study showed that $6 \%$ of Korean older adults were in the frail stage and $42.5 \%$ in the pre-frail stage. This was similar to the results of a study of Japanese older adults, which found $5.8 \%$ in the frail stage and $40.8 \%$ 
in the pre-frail stage [32], but indicated a significantly higher percentage of frail older adults compared to another study in Greece, where $1.5 \%$ of older adults were in the frail stage [16]. According to previous studies, the percentage of transition from pre-frail to frail is high [33]. It is crucial to provide interventions that prevent the transition between stages of frailty, especially by identifying factors influencing the transition from the prefrail to the frail stage.

Social isolation was an influential factor in the worsening transition between the stages of frailty. These results are similar to those of previous studies [21-23,34]. However, previous studies had limitations in that the multidimensionality of social isolation was not considered and the influence on each step of transition between the stages of frailty could not be analyzed. The results of this study, that the transition from the robust stage to pre-frail stage is influenced by both subjective and objective social isolation and the transition from the pre-frail to the frail stage is entirely influenced by objective social isolation, are new findings. Previous researchers have reported that subjective social isolation had a stronger correlation with emotional factors such as depressive symptoms and psychological distress than with physical health factors [25]. Therefore, in terms of frailty, objective social isolation may appear to be a more significant factor influencing the transition from the pre-frail to frail stage, compared to subjective social isolation. In addition, objective social isolation had a stronger influence on the transition from the pre-frail to frail stage than on the transition from the robust to pre-frail stage in this study. Objective social isolation indicates a reduced social network outside of cohabitants and reduced social interchange [24]. Thus, interventions should be provided to maintain social networks and promote social interchange among pre-frail older adults, with an emphasis on preventing the transition from the pre-frail to frail stage.

In this study, the level of objective social isolation increased with higher age, lower education level and household income, living alone, reduced sensory functions, ADL dependency, cognitive decline, and irregular exercise. As age increased, the number of people nearby that could help older adults decreases and contact with friends and acquaintances decreases. In addition, the social network with family, friends, and neighbors is reduced, leading to isolation [25]. Korean older adults used to live in a culture that valued family relationships, but the change toward nuclear family and different degrees of importance placed on the family by younger generations have gradually diminished the family-centered culture. This implies a need to prevent social isolation by reinforcing alternative social relations. This study's findings indicated that the probability of transition from pre-frail to frail was higher when the subjects were isolated from friends only or isolated from both friends and family than when they were isolated from family only. Thus, a stronger focus should be on the social network of older adults than on the family relationships.

Social isolation also varied substantially according to the socioeconomic position, with the highest isolation level observed among older adults living alone. These results were consistent with the findings in previous studies [35]. Therefore, to prevent social isolation that accelerates the transition to the frail stage, priority targets for interventions that help maintain existing social networks or help them connect with new social networks should be older adults with low socioeconomic status or older adults living alone.

ADL dependency, IADL dependency, reduced sensory functions, and cognitive decline showed a strong correlation with social isolation, a finding that was in line with previous studies [13,36, 37]. In Korea, people aged 65 and over who are in vulnerable groups on the basis of socioeconomic and health status are managed 
through the home visiting health service program of public health centers [38]. Approximately $9 \%$ of older people receive those services [39]. Several European countries and Japan have provided a preventive home visit service for older adults universally [40] and its effectiveness has been reported in several studies [41, 42]. Home visit nurses can regularly visit older adults living in the community to check their health status, monitor social networks, and provide interventions to maintain social networks by screening individuals at risk of social isolation [43]. Therefore, there is a need to universally provide the home visit health service in public health centers to older adults in South Korea.

Furthermore, factors influencing the transition from the pre-frail to frail stage were found to differ from those influencing the transition from the robust to pre-frail stage. Notably, hearing sensory and regular exercise were the two most important factors. First, the development of the pre-frail stage was influenced by reduced visual and auditory sensory functions. This result was consistent with that of a previous study [18]. The decline in hearing, in particular, was shown to be a risk factor in the transition from the pre-frail to frail stage. A common symptom among older adults is reduced hearing ability [43], which poses a challenge to communication and social interactions with others, leading to increased social isolation [44, 45]. Likewise, in this study, older adults with reduced hearing and those with normal hearing showed a difference in subjective and objective social isolation. Therefore, to prevent the worsening transition stages of frailty, regular hearing checkups in older adults appear to be especially important. Concomitantly, older adults with reduced hearing should be provided with an assistive device to help them proactively maintain their hearing sensory [45].

Second, older adults with irregular exercise showed a higher probability of transition from the pre-frail to frail stage than those who were exercising regularly This was consistent with other studies. Those studies suggested that regular exercise is a protective factor against frailty [17] and an effective intervention to prevent frailty [46]. Moreover, the combination of reduced physical activity and social isolation further increases the probability of transition to the frail stage [47]. In this study, likewise, the correlation between regular exercise and social isolation was high. Therefore, the transition from the pre-frail to frail stage may be prevented by developing a way to encourage the elderly to practice physical activity regularly within new or existing social networks. This study showed that different factors influenced each step of the transition between the stages of frailty. These results suggest that researchers should consider differences in factors that influence the stages of frailty among Korean older adults.

However, this study also has the following limitations. First, it was conducted as a cross-sectional study. Thus, a longitudinal study should be conducted to investigate the transitions among the stages of frailty. Second, while a cognitive decline was shown to be an important factor in previous studies, it was found not to influence the transition between the stages of frailty in this study. This may be because the data analyzed in this study were obtained from community residents with an insufficient number of older adults with cognitive decline.

\section{Conclusions}

This study investigated the factors influencing the transition between stages of frailty in Korean older adults, focusing on social isolation. The results showed that, while sociodemographic characteristics, health-related characteristics, and social isolation influenced the development of the pre-frail stage, objective social

Page $15 / 20$ 
isolation, reduced hearing, and lack of regular exercise had an influence on the transition from the pre-frail to frail stage. Based on the findings in this study, we suggest follows. First, interventions should be developed by frailty stages. Notably, for individuals in the pre-frail stage, support to help them maintain their relationships with family, relatives, and friends, in addition to their cohabitants, should be provided. Second, sensory function, especially hearing function should be maintained which plays an important role in social relationships. Regular hearing checkups should be performed and assistive devices should be actively provided to help older adults maintain their hearing sensory function. Third, Regular exercise was a critical factor influencing the transition from the pre-frail to frail stage. Therefore, older adults at each step of the transition between frailty stages should be encouraged to exercise regularly. Useful resources and support should be made available to help the older adults maintain their social network and exercise regularly in the pre-frail stage. Lastly, a cohort study should be conducted to identify the factors influencing each step of the transition between stages of frailty. An intervention study should also be conducted on older adults in the prefrail stage to verify the effects on delaying the transition to the frail stage.

\section{Abbreviations}

ADL: Activities of Daily Living

BMI: body mass index

Cl: Confidence Interval

IADL: Instrumental Activities of Daily Living

IRB: Institutional Review Board

M: mean

NSOK: National Survey of Older Koreans

OR: Odds Ratio

SD: standard deviation

\section{Declarations}

\section{Availability of data and materials}

The datasets used and/or analyzed during the current study are available from the corresponding author on reasonable request.

\section{Authors' Contribution}

SYH, HYJ, and YK were responsible for the study conception and design. SYH, HYJ, and YK were responsible for the drafting of the manuscript. SYH and YK performed the data analysis and HYJ provided statistical 
expertise. SYH provided administrative, technical or material support. SYH and YK supervised the study and SYH wrote the first draft. All authors read and approved the final manuscript.

\section{Ethical approval and consent to participate}

This study was conducted in accordance with the Declaration of Helsinki and was approved by the institutional review board (IRB) of Gachon University (IRB No.1044396-202102-HR-030-01). We were provided the data from the public data portal (data.go.kr) after the institution's application and subsequent approval. Written consent was waived since the data set of the National Survey of Older Koreans was completely anonymized under strict confidentiality guidelines.

\section{Consent for publication}

Not applicable

\section{Competing interests}

We have no conflict of interest to disclose.

\section{Funding}

We did not receive any funding or support from any agency.

\section{Acknowledgements}

None declared

\section{References}

1. Vermeiren S, Vella-Azzopardi R, Beckwee D, Habbig AK, Scafoglieri A, Jansen B, et al. Frailty and the prediction of negative health outcomes: a meta-analysis. J Am Med Dir Assoc. 2016;17(12):1163.e1-e17. doi:10.1016/j.jamda.2016.09.010

2. Jung HW, Kim SW, Ahn S, Lim JY, Han JW, Kim TH, et al. Prevalence and outcomes of frailty in Korean elderly population: comparisons of a multidimensional frailty index with two phenotype models. PloS one. 2014; 9(2):e87958.

3. Fried LP, Tangen CM, Walston J, Newman AB, Hirsch C, Gottdiener J, et al. Frailty in older adults: evidence for a phenotype. J Gerontol A Biol Sci Med Sci. 2001;56(3):M146-57. doi:10.1093/gerona/56.3.M146

4. Ahmed N, Mandel R, Fain MJ. Frailty: An emerging geriatric syndrome. Am JMed. 2007;120(9):748-53. doi:10.1016/j.amjmed.2006.10.018 
5. Park B, Lee Y. Integrative approach to elderly frailty. Korean J Fam Med. 2010;31:747-54. doi: 10.4082/kjfm.2010.31.10.747

6. Nagai K, Tamaki K, Kusunoki H, Wada Y, Tsuji S, Itoh M, et al. Physical frailty predicts the development of social frailty: a prospective cohort study. BMC geriatrics. 2020;20(1):1-8.

7. Abizanda P, Romero L, Sanchez-Jurado PM, Martinez-Reig M, Alfonso-Silguero SA, Rodriguez-Manas L. Age, frailty, disability, institutionalization, multimorbidity or comorbidity. Which are the main targets in older adults?. J Nutr Health Aging. 2014;18(6):622-7.

8. Zhang Y, Xu XJ, Lian TY, Huang LF, Zeng JM, Liang DM, et al. Development of frailty subtypes and their associated risk factors among the community-dwelling elderly population. Aging (Albany NY), 2020;12(2):1128-40. doi: 10.18632/aging.102671

9. Chang YW, Chen WL, Lin FG, Fang WH, Yen MY, Hsieh CC, et al. Frailty and its impact on health-related quality of life: A cross-sectional study on elder community-dwelling preventive health service users. PloS One. 2012;7(5):e38079. doi:10.1371/journal.pone.0038079

10. Yang F, Chen QW. Evaluation of frailty and influencing factors in old people in hospital institution: Evidence for a phenotype of frailty. Medicine. 2018;97(3). doi: 10.1097/MD.0000000000009634

11. Shi SM, Olivieri-Mui B, McCarthy EP, Kim DH. Changes in a frailty index and association with mortality.J Am Geriatr Soc. 2020;69(4):1057-62. doi:10.1111/jgs.17002

12. Niederstrasser NG, Rogers NT, Bandelow S. Determinants of frailty development and progression using a multidimensional frailty index: Evidence from the English Longitudinal Study of Ageing.PloS one. 2019;14(10):e0223799. doi:10.1371/journal.pone.0223799

13. Jung HW, Yoo HJ, Park SY, Kim SW, Choi JY, Yoon SJ, et al. The Korean version of the FRAIL scale: clinical feasibility and validity of assessing the frailty status of Korean elderly. Korean J Intern Med 2016;31:594600. doi:10.3904/kjim.2014.331

14. Martin FC, O'Halloran AM. Tools for assessing frailty in older people: general concepts. Adv Exp Med Biol. 2020;1216:9-19. Doi:10.1007/978-3-030-33330-0_2

15. van Kan, G. A., Rolland, Y. M., Morley, J. E., \& Vellas, B.. Frailty: toward a clinical definition. Journal of the American Medical Directors Association, 2008;9(2): 71-72. doi: 10.1016/j.jamda.2007.11.005.

16. Ntanasi E, Yannakoulia M, Mourtzi N, Vlachos GS, Kosmidis MH, Anastasiou CA, et al. Prevalence and risk factors of frailty in a community-dwelling population: the HELIAD study. J Aging Health. 2020;32(1):1424. doi: $10.1177 / 0898264318801735$

17. Zhang Y, Xu XJ, Lian TY, Huang LF, Zeng JM, Liang DM, et al. Development of frailty subtypes and their associated risk factors among the community-dwelling elderly population. Aging (Albany NY). 2020;12(2):1128-40. doi: 10.18632/aging.102671

18. Trevisan C, Veronese N, Maggi S, Baggio G, Toffanello ED, Zambon S, et al. Factors influencing transitions between frailty states in elderly adults: The Progetto Veneto Anziani Longitudinal Study.JAm Geriatr Soc. 2017;65(1):179-84. doi: 10.1111/jgs. 14515

19. Yoon DH, Hwang SS, Lee DW, Lee CG, Song W. Physical frailty and cognitive functioning in Korea rural community-dwelling older adults.J Clin Med.2018;7(11): 405. doi:10.3390/jcm7110405 
20. Furtado GE, Caldo A, Rieping T, Filaire E, Hogervorst E, Teixeira AMB, et al. Physical frailty and cognitive status over-60 age populations: a systematic review with meta-analysis.Arch Gerontol Geriatr. 2018;78:240-48.

21. Hoogendijk EO, Suanet B, Dent E, Deeg DJ, Aartsen MJ. Adverse effects of frailty on social functioning in older adults: results from the Longitudinal Aging Study Amsterdam. Maturitas. 2016;83:45-50. doi: 10.1016/j.maturitas.2015.09.002

22. Makizako H, Shimada H, Doi T, Tsutsumimoto K, Hotta R, Nakakubo S, et al. Social frailty leads to the development of physical frailty among physically non-frail adults: a four-year follow-up longitudinal cohort study. Int J Environ Res Public Health. 2018;15(3):490. doi:10.3390/ijerph15030490

23. Maltby J, Hunt SA, Ohinata A, Palmer E, Conroy S. Frailty and social isolation: comparing the relationship between frailty and unidimensional and multifactorial models of social isolation. J Aging Health. 2020;32(10):1297-308. doi: 10.1177/0898264320923245

24. Taylor RJ, Taylor HO, Chatters LM. Social isolation from extended family members and friends among African Americans: findings from a national survey. J Fam Soc Work. 2016;19:443-61.

25. Taylor HO, Taylor RJ, Nguyen AW, Chatters L. Social isolation, depression, and psychological distress among older adults.J Aging Health. 2018;30(2):229-46. doi:10.1177/0898264316673511.

26. Carstensen LL, Isaacowitz DM, Charles ST. Taking time seriously: a theory of socioemotional selectivity. Am Psychol. 1999; 54(3):165-81.

27. McPherson M, Smith-Lovin L, Brashears ME. Social isolation in America: changes in core discussion networks over two decades. Am Sociol Rev. 2006;71(3):353-75.

28. Korea Institute for Health and Social Affairs. 2017 National Survey of Older Koreans.; Ministry of Health and Welfare: Sejong, Korea, 2017; pp. 27-39.

29. Morley JE, Malmstrom TK, Miller DK. A simple frailty questionnaire (FRAIL) predicts outcomes in middle aged African Americans. J Nutr Health Aging. 2012;16:601-8. doi:10.1007/s12603-012-0084-2

30. Won CW, Yang KY, Rho YG, Kim SY, Lee EJ, Yoon JL, et al. The development of Korean Activities of Daily Living (K-ADL) and Korean Instrumental Activities of Daily Living (K-IADL) scale. J Korean Geriatr Soc. 2002;6:107-20.

31. Kim GW, Kim MH, Kim BG, Kim JR, Kim TH, Moon SW, et al. Standardization of diagnostic tools for dementia. Research Report. Gyeonggido, Seongnam: Bundang Seoul National University Hospital; 2009 December. Report No.: 11-1351000-000589-01.

32. Chen S, Honda T, Narazaki K, Chen T, Kishimoto H, Haeuchi Y, et al. Physical frailty is associated with longitudinal decline in global cognitive function in non-demented older adults: a prospective study. J Nutr Health Aging. 2018;22(1):82-8. doi: 10.1007/s12603-017-0924-1

33. Cheong CY, Nyunt MSZ, Gao Q, Gwee X, Choo RWM, Yap KB, et al. Risk factors of progression to frailty: findings from the Singapore Longitudinal Ageing Study. J Nutr Health Aging. 2020;24(1):98-106. doi:10.1007/s12603-019-1277-8

34. Jarach CM, Tettamanti M, Nobili A, D'avanzo B. Social isolation and loneliness as related to progression and reversion of frailty in the Survey of Health Aging Retirement in Europe (SHARE). Age Ageing. 2021;50(1):258-62. doi: 10.1093/ageing/afaa168 
35. Macdonald SJ, Nixon J, Deacon L. Loneliness in the city: examining socioeconomics, loneliness and poor health in the North East of England. Public Health. 2018:165;88-94. doi: 10.1016/j.puhe.2018.09.003

36. Coyle CE, Steinman BA, Chen J. Visual acuity and self-reported vision status. J Aging Health. 2017;29(1):128-48. doi:10.1177/0898264315624909

37. Ejiri M, Kawai H, Ishii K, Oka K, Obuchi S. Predictors of older adults' objectively measured social isolation: A systematic review of observational studies. Arch Gerontol Geriatr. 2021;94:104357. doi: 10.1016/j.archger.2021.104357

38. Ministry of Health and Welfare, Korea Health Promotion Institute. 2019 Guideline for visiting health care. Seoul: Korea Health Promotion Institute; 2019. pp. 42.

39. Korea Health Promotion Institute. 2018 Study on the status and direction of development of visiting health care programs. Seoul: Korea Health Promotion Institute, 2020. Report No. 04-2020-003-01.

40. Stijnen MMN, Jansen MWJ, Vrijhoef HJM, Duimel-Peeters IGP. Development of a home visitation programme for the early detection of health problems in potentially frail community-dewelling older people by general practices. Eur J Ageing. 2012;10:49-60. doi: 10.1007/s10433-012-0251-7

41. Liimatta $\mathrm{H}$, Lampela $\mathrm{P}$, Laitinen-Parkkonen P, Pitkala KH. Effects of preventive home visits on healthrelated quality-of-life and mortality in home-dwelling older adults. Scand J Prim Health Care. 2019;37(1):90-7. doi: 10.1080/02813432.2019.1569372

42. Kono A, Kanaya Y, Fujita T, Tsumura C, Kondo T, Kushiyama K, et al. Effects of a preventive home visit program in ambulatory frail older people: a randomized controlled trial. J Gerontol A Biol Sci Med Sci. 2012 Mar;67(3):302-9. doi: 10.1093/gerona/glr176

43. Lee DO, Chin YR. The follow-up study of changes in frailty in elderly receiving home health care of the public health center. J Korean Acad Community Health Nurs. 2019;30(4):528-38. doi: 10.12799/jkachn.2019.30.4.528

44. Shukla A, Harper M, Pedersen E, Goman A, Suen JJ, Price C, et al. Hearing loss, loneliness, and social isolation: a systematic review. Otolaryngol. Head Neck Surg. 2020;162(5), 622-33. doi: $10.1177 / 0194599820910377$

45. Campos JL, Launer S. (2020). From healthy hearing to healthy living: a holistic approach. Ear Hear. 2020;41 Suppl 1:99S-106S. doi: 10.1097/AUD.0000000000000931.

46. Fernández-García Ál, Gómez-Cabello A, Moradell A, Navarrete-Villanueva D, Pérez-Gómez J, Ara I, et al. How to improve the functional capacity of frail and pre-frail elderly people? Health, nutritional status and exercise intervention. the EXERNET-Elder 3.0 project. Sustainability. 2020;12(15):6246. doi:10.3390/su12156246

47. Merchant RA, Morley JE, Izquierdo M. Exercise, aging and frailty: guidelines for increasing function. J Nutr Health Aging. 2021;25:1-5. 